\title{
Tau is central in the genetic Alzheimer- frontotemporal dementia spectrum
}

\author{
Bart Dermaut, Samir Kumar-Singh, Rosa Rademakers, Jessie Theuns, \\ Marc Cruts and Christine Van Broeckhoven
}

Department of Molecular Genetics (VIB 8), Flanders Interuniversity Institute for Biotechnology, Neurodegenerative Brain Diseases Group, University of Antwerp, Campus Drie Eiken, Universiteitsplein 1, B-2610 Antwerpen, Belgium

In contrast to the common and genetically complex senile form of Alzheimer's disease (AD), the molecular genetic dissection of inherited presenile dementias has given important mechanistic insights into the pathogenesis of degenerative brain disease. Here, we focus on recent genotype-phenotype correlative studies in presenile $A D$ and the frontotemporal dementia (FTD) complex of disorders. Together, these studies suggest that $A D$ and FTD are linked in a genetic spectrum of presenile degenerative brain disorders in which tau appears to be the central player.

\section{Introduction}

Through the genetic dissection of Alzheimer's disease (AD), we hope to increase our mechanistic understanding of this prevalent and untreatable disorder in which intraand extraneuronal protein aggregates (known as tau tangles and amyloid plaques, respectively) accumulate in the degenerating brain. In particular, genetic studies of the rare and genetically simple early onset or presenile (onset before 65 years of age) forms of $\mathrm{AD}$ have led to the identification of several single gene lesions in the amyloid precursor protein (APP) [1] and the presenilins (PS) [2,3]. In addition, mutations in the gene encoding the microtubule associated protein tau (MAPT) cause autosomal dominant forms of frontotemporal dementia (FTD) [4,5], a degenerative brain disease that has overlapping features with $\mathrm{AD}$ (Box 1). Collectively, the discovery of these mutations has been highly instructive in delineating our current mechanistic understanding of $\mathrm{AD}$ and FTD. For detailed and up-to-date information on $A P P, P S$ and $M A P T$ mutations, please see our interactive $\mathrm{AD}$ and FTD mutation database (http://www.molgen.ua.ac.be/ ADMutations).

By contrast, similar to most frequent diseases, the genetic architecture of the common late-onset or senile form of $\mathrm{AD}$ (age of onset above 65 years; 90\% of all $\mathrm{AD}$ patients) is complex. So far the $\varepsilon 4$ allele of the gene encoding apolipoprotein E (APOE) is the only wellestablished genetic risk-factor for late-onset $\mathrm{AD}$, but the underlying mechanism remains poorly defined [6-8]. In addition, several studies have investigated numerous

Corresponding author: Van Broeckhoven, C. (christine.vanbroeckhoven@ua. ac.be)

Available online 10 October 2005 candidate loci and genes, but none of these has reached the established position of $A P O E$. The current status of potential candidate risk genes and loci has been extensively reviewed recently [9]; for an update, see the Alzheimer's Research Forum genetic database, 'Alzgene' (http://www.alzgene.org).

In this review, we emphasize that careful genotypeproteotype-phenotype correlative studies, including molecular genetic, biochemical, neuropathological and clinical investigations of inherited presenile forms of AD and FTD, are instrumental in defining the complete phenotypic spectrum associated with mutations in $A P P, P S$ and $M A P T$ and will significantly advance our biological understanding of these diseases. More specifically, we review recent exciting evidence that $\mathrm{AD}$-causing $P S$ mutations have intrinsic loss-of-function properties and that PS lossof-function has a role in FTD and amyloid-independent

\footnotetext{
Glossary

Cerebral amyloid angiopathy (CAA): CAA is a histopathological term referring to the deposition of $A \beta$ in the blood vessel walls of the brain (angiopathy), thereby predisposing individuals to brain bleeding (haemorrhagic strokes). Severe CAA and haemorrhagic stroke are characteristic features of mutations affecting the $\alpha$-secretase-cleavage site of APP. CAA is a frequent observation in $A D$ brains but usually does not result in strokes.

Progressive supranuclear palsy (PSP): PSP is a sporadic neurodegenerative Parkinson-like movement disorder. PSP is characterized by vertical eye movement disturbances, rigidity (resistance to imposed movement), severe gait and balance problems and FTD-like dementia later in the disease course. Abundant tau pathology in the form of tau tangles characterizes PSP neuropathologically. PSP is part of the FTD spectrum of disorders.

Corticobasal degeneration (CBD): $\mathrm{CBD}$ is a sporadic neurodegenerative Parkinson-like movement disorder and has many clinical overlapping features with PSP. The major difference with PSP is a marked asymmetry of the symptoms at onset. CBD is further characterized by impaired balance and abnormal muscle postures of the limb (dystonia). FTD-like dementia is also often observed later in the disease course. Abundant tau pathology in the form of fine filaments characterizes CBD neuropathologically. CBD is part of the FTD spectrum of disorders.

Spastic paraparesis: Spastic paraparesis is characterized by insidiously progressive bilateral lower extremity weakness and spasticity. The primary pathology in spastic paraparesis is degeneration of the distal ends of the long spinal axons of the cortical motor neurons. Some PS mutations are associated with $A D$ and spastic paraparesis.

Pick bodies: Pick bodies are tau-postive cytoplasmic neuronal inclusion and are the pathological hallmark lesions of Pick's disease, a neuropathological subtype of tau-postive FTD. Pick bodies are not related to the Lewy bodies observed in Parkinson's disease, which are tau-negative and $\alpha$-synucleinpositive.

Niemann-Pick disease type $\mathbf{C}$ (NPC): NPC is a recessive lysosomal disorder of childhood probably caused by lysosomal storage of cholesterol. In NPC the neuronal degeneration is characterized by prominent tau pathology in the form of AD-like tau tangles. Mild adult-onset forms of this disease exist and then present as psychatric disease and presenile dementia.
} 


\section{Box 1. Overlap between presenile AD and FTD}

FTD and $A D$ are primary degenerative dementias, meaning that a gradual loss of neurons is responsible for the progressive brain dysfunction. Within the group of presenile dementias (onset $<65$ years of age), FTD is the second most common form of neurodegenerative dementia after $A D$. Clinically, $A D$ is primarily a disease of memory and cognition caused by a more genereralized brain atrophy, starting in the medial temporal lobe. The hallmarks of FTD are behavior and/or language dysfunction caused by a more focal degeneration mainly affecting the frontal and temporal brain regions. Nevertheless and despite the existence of usefud clinical diagnostic criteria for both disorders, the distinction between

neurodegeneration. In addition, we review recent studies that further confirm the primary genetic role of $M A P T$ in the FTD complex of disorders and give an update on a novel genetic form of FTD linked to the MAPT region with the striking absence of demonstrable MAPT mutations and tau pathology.

\section{Defining the extremes: APP versus MAPT phenotypes APP disorders: the 'amyloidocentric' view}

Initial genetic linkage studies mainly targeted chromosome 21 , because the brains of almost all middle-aged Down syndrome patients, who have a trisomy of chromosome 21, contain the neuropathological hallmarks of $\mathrm{AD}$. The underlying hypothesis was that in Down syndrome patients, $\mathrm{AD}$ is caused by over-representation of a gene on chromosome 21 as a result of the trisomy 21 , whereas in $\mathrm{AD}$, a mutation in that gene might result in the production of an abnormal protein or the overproduction of a normal protein $[10,11]$. Since the identification of the first ADcausing mutation in $A P P$ at $21 \mathrm{q} 21$ [1], 18 different causative $A P P$ mutations have now been reported (Figure 1; $\mathrm{AD}$ and FTD Mutation Database: http://www. molgen.ua.ac.be/ADMutations). Strikingly, all of these mutations cluster at, or are near to, sites within APP that are normally cleaved by proteases called the $\alpha$-, $\beta$ - and $\gamma$-secretases. These enzymatic activities regulate the metabolism of APP including the generation of the A $\beta$ peptide, which is the major constituent of the amyloid plaques. Interestingly, the phenotypic outcome of these mutations is strongly dependent on which cleavage site is mutated. For example, mutations affecting the $\alpha$-cleavage site promote the self-aggregation of mutated $A \beta$ peptides, leading to severe amyloid deposition within the cerebral vessel walls or cerebral amyloid angiopathy (CAA) and hence strongly predispose affected individuals to haemorrhagic strokes (Figure 2). However, with the exception of the Dutch APP E693Q mutation, which causes a pure CAA-related haemorrhagic stroke phenotype [12,13], these mutations at the $\alpha$-cleavage site also lead to the deposition of amyloid plaques and tau tangles in the brain parenchyma in addition to CAA, which together result in a combined neurodegenerative and haemorrhagic stroke disorder as illustrated by the Flemish APP A692G mutation $[14,15]$. However, mutations affecting the $\beta$ [16] and $\gamma$-secretase sites [1] favor the release of amyloidogenic $A \beta 42$ from its precursor and mostly result in typical $\mathrm{AD}$ phenotypes characterized by amyloid plaques and tau tangles with CAA being less prominent. presenile AD and FTD can be difficult, especially in presenile cases, which often have an atypical presentation. In particular, the inherited forms of FTD have an extremely broad phenotype ranging from typical FTD to cases where parkinsonism, amyotrophy or ADlike memory impairment are the major disabling features. In addition, focal variants of $A D$, where memory loss is not a prominent early feature, are relatively common in a presenile setting and can mimic FTD. Because deposition of tau protein aggregates in affected brain regions is a common feature in $A D$ and tau-positive FTD, it is likely that both disorders share a common pathogenetic mechanism.

Interestingly, the Austrian APP T714I mutation, which is located at the $\gamma$-secretase-cleavage site of APP, results in an extremely aggressive $\mathrm{AD}$ phenotype with an onset age of $\sim 35$ years and is characterized by tau tangles and extensive deposition of nonfibrillar 'cotton wool' amyloid plaques [17].

Together, genotype-phenotype correlation studies of APP mutations strongly implicate various forms of $A \beta$

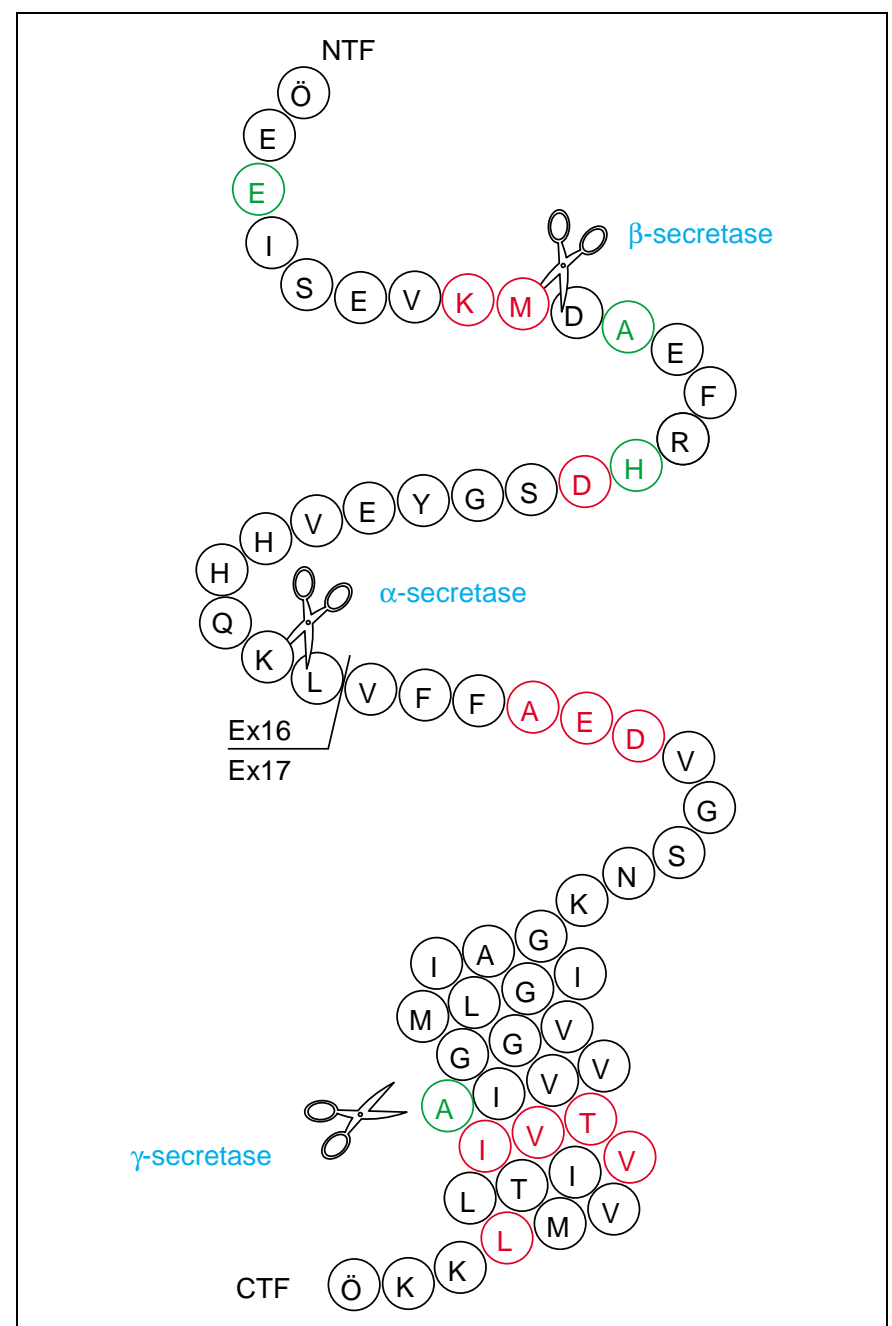

Figure 1. APP mutations. Schematic representation of the protein sequence encoded by exons 16 and 17 of APP. Each circle represents an amino acid; those with pathogenic missense mutations are shown in red and those with nonpathogenic missense mutations are in green. Pathogenic mutations are always at or close to sites that are cleaved by the $\alpha$-, $\beta$ - or $\gamma$-secretases. The figure was adapted with permission from the Alzheimer Research Forum (http://www.alzforum.org/) and can also be found at our AD and FTD mutation database available at http:// www.molgen.ua.ac.be/ADMutations. 

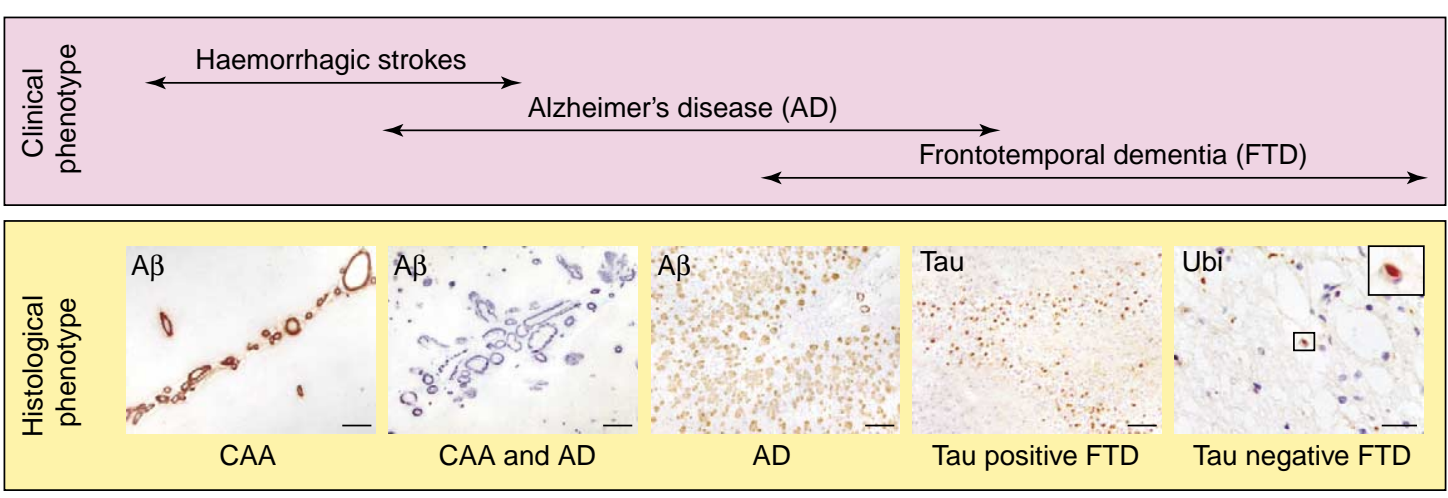

\begin{tabular}{|c|c|c|c|c|c|c|}
\hline \multirow{3}{*}{ 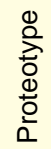 } & $A \beta$ & +++ & +++ & +++ & - & - \\
\hline & Tau & \pm & ++ & +++ & ++ & - \\
\hline & Ubi & \pm & ++ & +++ & ++ & $(+)$ \\
\hline
\end{tabular}

$\sum_{\substack{0 \\ 0}}^{\substack{0 \\ 0}}$

$\overline{T R E N D S}$ in Genetics

Figure 2. The genetic AD-FTD spectrum of degenerative brain disease This figure integrates the genotype-proteotype-phenotype correlations and suggests the existence of a genetically interconnected spectrum of AD and FTD disorders. The FTD complex of disorders includes PSP and CBD, sporadic disorders with prominent parkinsonism neuropathologically characterized by tau deposits. Throughout the spectrum the involvement of tau either histopathologically or genetically appears to be a constant characteristic. Images in the histological phenotype panel represent prototypical examples of neuropathological dementia subtypes. CAA: A $\beta$-positive blood vessels in the temporal region of a patient with the Dutch APP E6930 mutation; CAA and AD: A $\beta$-positive blood vessels and dense cored plaques in the temporal region of a patient with the Flemish APP A692G mutation; AD: A 3 -positive dense cored plaques in the temporal region of a patient with the PS1 I143T mutation; Tau positive FTD: tau-positive Pick bodies in the hippocampus of a patient with the PS1 G183V mutation; Tau negative FTD: cytoplasmic and intranuclear ubiquitin (Ubi) positive inclusions in the temporal region of a patient of a family linked to the MAPT locus but without MAPT mutations. Scale bars represent $200 \mu \mathrm{m}$ except in Tau negative FTD, where the scale bare corresponds to $50 \mu \mathrm{m}$. In the proteotype panel: +++ indicates highly abundant; ++ indicates abundant; $(+)$ indicates present but inconsistently; \pm indicates sometimes present and means absent. In the genotype panel: arrows correspond to clearly established genotype-phenotype links, and dashed arrows correspond to probable links that need confirmation.

deposition, ranging from vascular CAA and fibrillar corecontaining plaques to non-fibrillar 'cotton wool' plaques, as an essential characteristic of $\mathrm{AD}$ (Figure 2). Interestingly, our own morphological studies of APP mutations in human and mouse have strongly implicated the vascular system in the formation of core-containing amyloid plaques suggesting that vascular damage might be an important contributing factor to $\mathrm{AD}$ pathogenesis $[15,18]$. Importantly and with the possible exception of the Dutch APP mutation, tau deposits in the form of tangles are a consistent but downstream consequence throughout the APP spectrum of disorders.

\section{MAPT disorders: the 'tauocentric' view}

In 1998, the first mutations in $M A P T$ causing autosomal dominant FTD were identified [4,5] and 40 different causative MAPT mutations have now been reported (Figure 3 and FTD Mutation Database: http://www. molgen.ua.ac.be/FTDMutations) [19]. Interestingly, nearly all mutations are located in the C-terminus of the protein and include missense, silent and intronic variations in addition to two single codon deletions clustered in or near the microtubule-binding domains. Importantly and in sharp contrast to APP phenotypes, MAPT disorders are neuropathologically characterized by absence of $A \beta$ deposits but share with $\mathrm{AD}$ the invariable presence of different forms of tau aggregates and are therefore called pure tauopathies [20] (Figure 2). Clinically, MAPT mutations most typically present with FTD. However, the spectrum of MAPT disease is surprisingly wide and ranges from phenotypes in which FTD is accompanied by severe parkinsonism and motor neuron disease to degenerative disorders that are, as in the case of the MAPT R406W mutation, clinically hardly distinguishable from $\mathrm{AD}[21,22]$.

More recently, several autosomal dominant FTD families have been described that lack visible tau positive lesions but are still conclusively linked to a chromosomal region that contains $M A P T$ [23-25]. In these families, the neuropathological phenotype has been described as either 'dementia lacking distinctive histopathology' [23] or 'FTD with tau-negative and ubiquitin-positive inclusions' $[24,25]$, although it is currently unclear if these represent distinct disease entities or are pathological manifestations of the same primary defect. Strikingly however and consistent with the absence of tau-positive lesions, no causative MAPT mutations have been found in these families, despite extensive sequencing of the whole genomic MAPT locus [26]. Although this might be explained by a defect in another gene in close proximity to MAPT [24], others have suggested that this FTD subtype is a 'no tau tauopathy' caused by a primary tau 
(a)

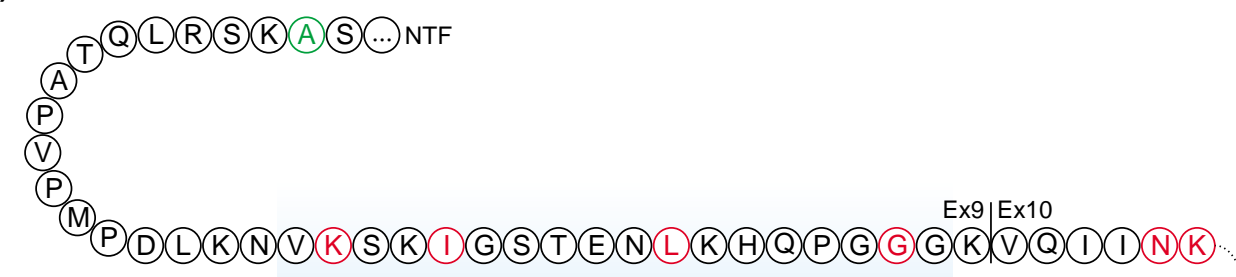

Ex10 Ex11

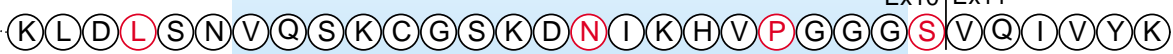

Ex11|Ex12

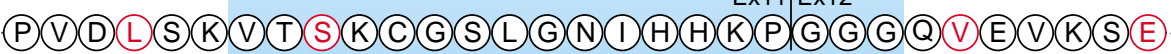

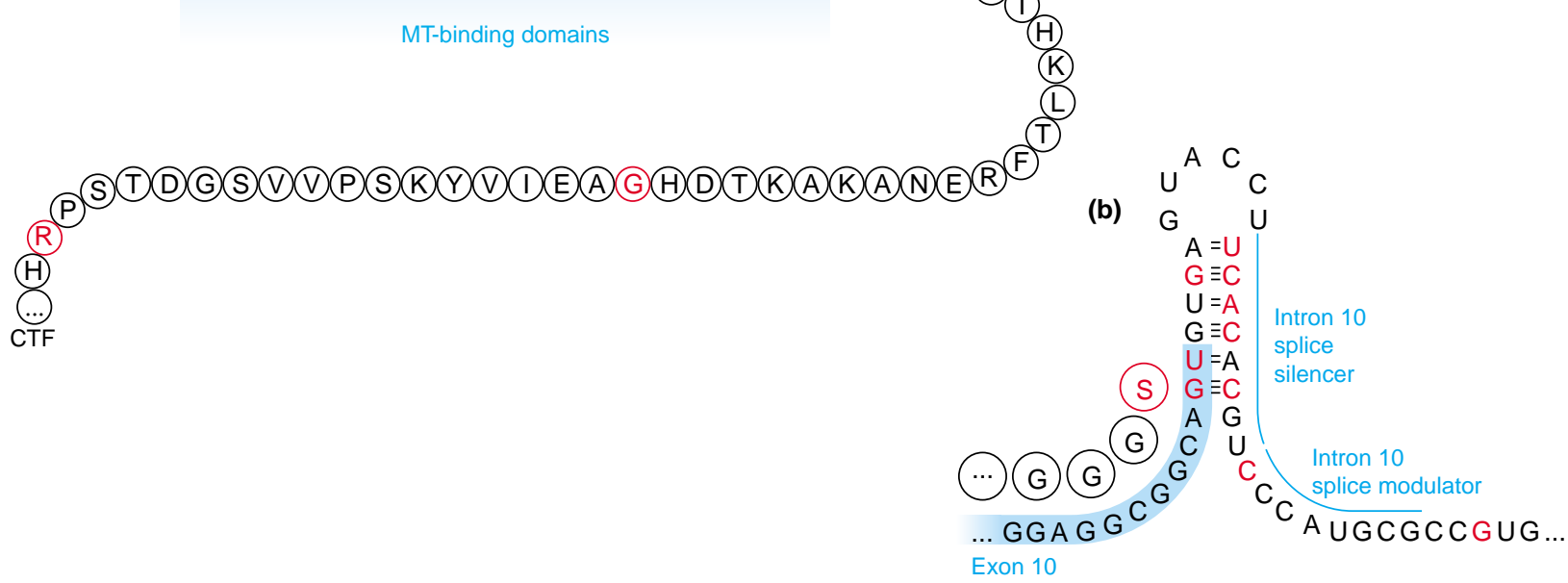

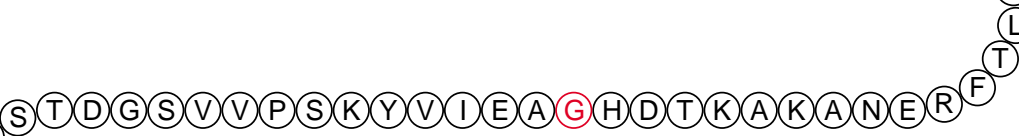

Ex12 Ex13

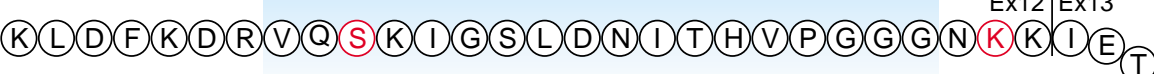

TRENDS in Genetics

Figure 3. MAPT mutations (a) Schematic representation of MAPT exons nine to 13 encoding the four microtubule-binding domains and inter-repeat regions of the tau protein.

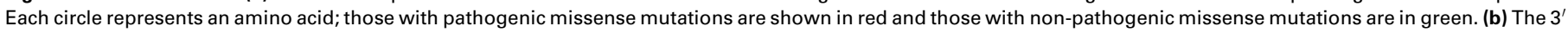

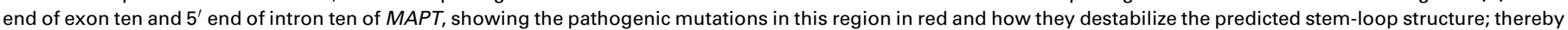

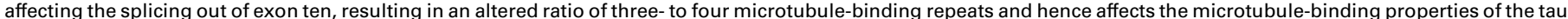

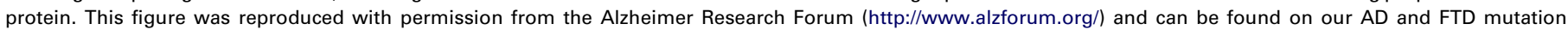
database: http://www.molgen.ua.ac.be/ADMutations.

defect that leads to loss of brain tau but not tau mRNA $[27,28]$. Nevertheless, this finding was not replicated and remains controversial [29]. In this context however, it is worthwhile mentioning that we and others have recently shown that MAPT along with several other genes are within a genomic $900-\mathrm{kb}$ region flanked by inverted lowcopy repeats (LCRs) that through non-allelic homologous recombination during primate evolution have induced a genomic inversion polymorphism called $\mathrm{H} 1$ and $\mathrm{H} 2$ $[30,26]$. These observations suggest that these LCRs render the $M A P T$ region susceptible to genomic rearrangements and that an as yet unidentified genomic mutation might be the cause of FTD in these families [31]. Moreover, absence of visible tauopathy does not exclude the possible genetic involvement of $M A P T$ - studies in Drosophila [32] and mouse models [33] have shown that tau-mediated neurodegeneration can be dissociated from visible pathologic tau aggregates. Because FTD without detectable tau pathology is a frequently observed neuropathological FTD subtype [34,35], the identification of the underlying gene defect in $M A P T$ or a neighboring gene is of great importance and will significantly contribute to our understanding of the neurodegenerative process in this type of FTD.

Although a causative role for MAPT in the degenerative process in these FTD families appears possible but remains unproven, a primary genetic role of $M A P T$ as a susceptibility gene in sporadic pure tauopathies called progressive supranuclear palsy (PSP) and corticobasal degeneration (CBD) is likely. PSP and CBD are sporadic disorders with prominent parkinsonism neuropathologically characterized by tau deposits and are part of the FTD complex of disorders [36]. Interestingly, homozygosity of $M A P T$ polymorphisms that segregate on the extended $\mathrm{H} 1$ haplotype are consistently overrepresented in patients with PSP [37,38] and CBD [39]. Although the genetic 
mechanism explaining this well-replicated association remains unresolved, in vitro studies have suggested that the MAPT H1 haplotype might be more efficient at driving $M A P T$ gene expression than the $\mathrm{H} 2$ haplotype [40]. To further understand the genetic mechanism explaining the association, we recently generated a high-density single nucleotide polymorphism (SNP) map by sequencing $138 \mathrm{~kb}$ of the genomic region of MAPT (R. Rademakers et al., personal communication) and identified a $22-\mathrm{kb}$ PSP-risk-containing regulatory region in the large intron preceding the first coding exon of $M A P T$, which was fully explained by one SNP, htSNP167, creating a transcription factor CP2 (TFCP2) binding site. Recently, Pittman et al. [41] published SNP haplotype association data from a PSP patient-control sample that overlapped with the extended American sample we used in our study and identified a $56.3-\mathrm{kb}$ risk-increasing interval that also contained htSNP167.

Together these data strongly indicate that MAPT, through a toxic gain-of-function mechanism, is capable of inducing neuronal death leading to a wide range of degenerative phenotypes that can be grouped under the FTD or Pick complex of degenerative brain disorders [36] (Figure 2). A toxic gain-of-function is also supported by several animal model studies in which overexpression of mutant and wild-type tau causes neurodegeneration [42,43,32].

\section{In between the extremes: PS disorders?}

Although the phenotypes induced by APP and MAPT lesions are strongly supportive for an 'amyloidocentric' and 'tauocentric' view of neurodegeneration, respectively, recent genetic evidence suggests that these pathways might converge at the level of PS (Figure 2).

First, strong evidence exists for a direct etiological link between PS and APP processing. Since the identification of mutations in PS [2,3], the most common cause of inherited presenile $\mathrm{AD}, 154$ different $P S$ mutations (144 in PS1 and ten in PS2), mostly of the missense type, have been identified (Figure 4 and AD Mutation Database: http://www.molgen.ua.ac.be/ADMutations). Similar to mutations at the $\beta$ - or $\gamma$-secretase-cleavage site, PS mutations generally result in typical $\mathrm{AD}$ phenotypes with amyloid plaques and tau tangles. In addition, $\mathrm{AD}$ causing $P S$ mutations increase the in vitro ratio of $\mathrm{A} \beta 42$ to $\mathrm{A} \beta 40$ ? [44], and it is now well established that PS is a core component if not the catalytic subunit of the multimeric $\gamma$-secretase $[45,46]$. In general, patients with PS1

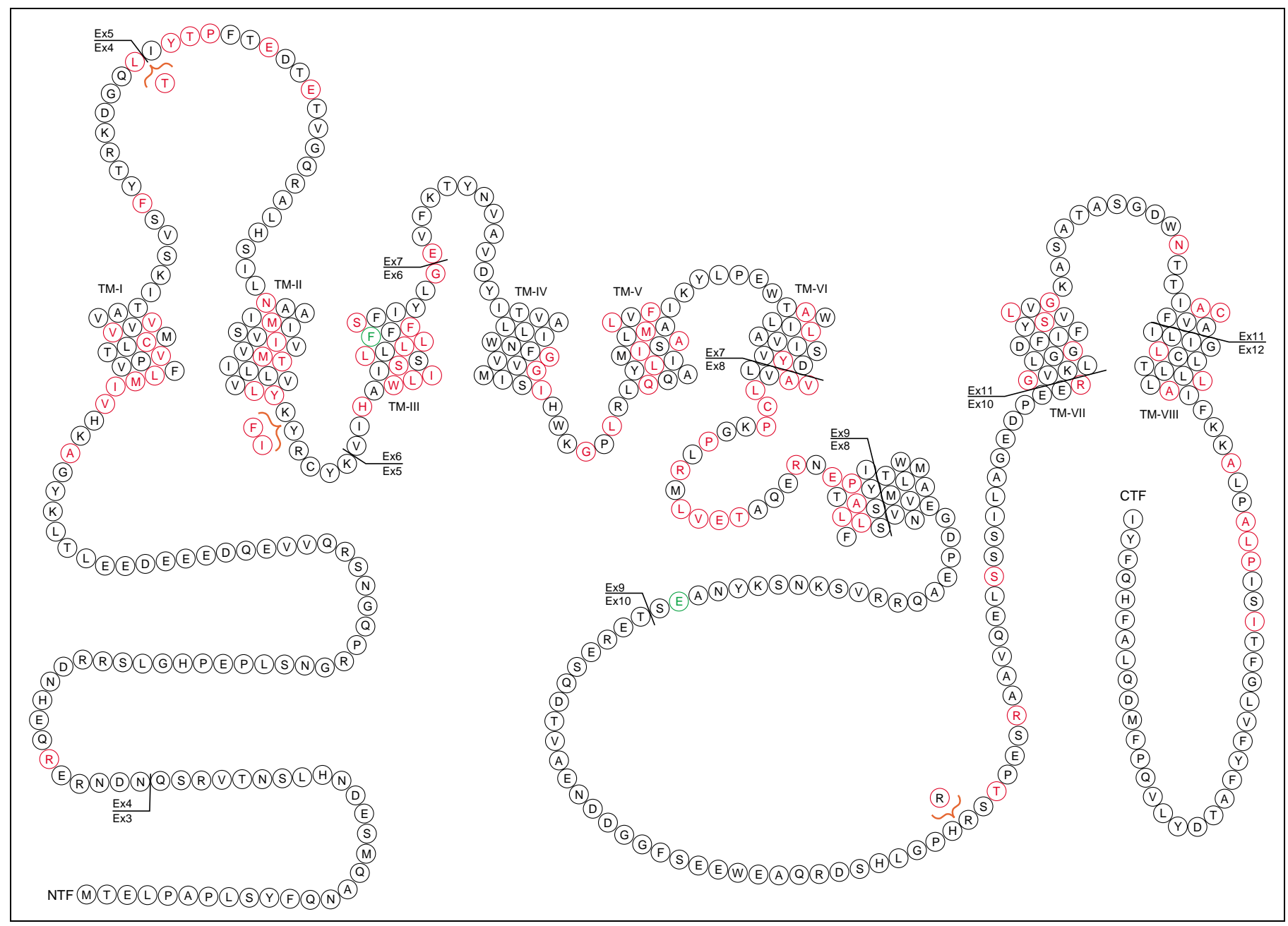

Figure 4. PS mutations. Schematic representation of PS1, the most frequently mutated gene in presenile AD. Each circle represents an amino acid; those with pathogenic

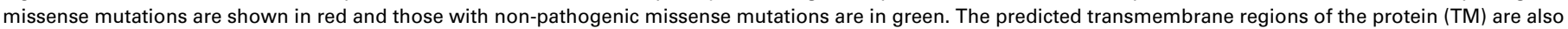

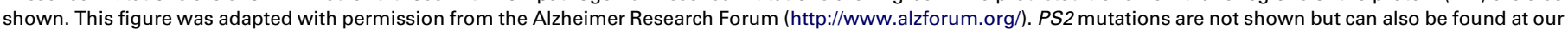
online AD and FTD mutation database available at http://www.molgen.ua.ac.be/ADMutations. 
mutations have a more aggressive disease course and earlier age of onset compared with those carrying PS2 mutations [47].

Interestingly, $P S$ mutations appear capable of inducing a relatively wide spectrum of clinical and neuropathological phenotypes. However, some PS mutations, particularly in cases in which the mutation lies beyond codon 200 , are associated with a prominent CAA [48], sometimes, even reminiscent of the Flemish APP A692G $\alpha$-secretase mutation [14] (e.g. the PS1 L282V mutation [49]). In contrast to mutations at the APP $\alpha$-secretase-cleavage site and with one reported exception [50], PS-related CAA is generally not sufficiently severe to cause haemorrhagic strokes. Another type of $P S$ mutations leads to 'variant' $\mathrm{AD}$, presenting with spastic paraparesis and diffuse 'cotton wool' plaques [51,52], similar to those observed in carriers of the Austrian APP mutation [17]. It was suggested that cases with 'variant' $\mathrm{AD}$ represent an aggressive subtype of $\mathrm{AD}$ because $P S$ mutations causing this condition lead to exceptionally high in vitro amyloid concentrations [53]. Nevertheless, although these PS mutations have interesting and unusual features, they are consistent with aberrant APP processing being the primary underlying mechanism in these disorders. Again, it is important to emphasize that all these PS mutations also lead to prominent tau pathology, albeit to a different extent. Unfortunately, no studies are available that, similar to CAA [48], have systematically addressed whether the severity of tau pathology is also determined by PS mutation position.

More recently, the phenotypic spectrum of PS mutations has been extended to FTD [54]. For example, PS1 L113P, G183V and insArg352 were identified in families with clinical FTD [55-57]. Interestingly, neuropathological examination in a patient with PS1 G183V revealed tauopathy in the form of Pick bodies and complete absence of amyloid plaque pathology [57]. Consistent with the idea that $P S$ mutations can produce Pick bodies [57], co-existent $\mathrm{AD}$ and Pick body pathology was observed in patients with PS1 M146L and A260V mutations [58,59].

Together, the phenotypic spectrum associated with $P S$ mutations appears to be broad, ranging from phenotypes strongly resembling the $A P P$ mutations at the $\alpha$-secretasecleavage site to FTD without A $\beta$ pathology (Figure 2).

\section{Do $A P P$ and $P S$ mutations have gain- or loss-of-function properties?}

An important but still unresolved issue, with respect to clinical $A P P$ and $P S$ mutations, is whether they represent gain- or loss-of-function alleles. The answer to this question is important not only from a mechanistic point of view, but also with respect to the development of therapeutics targeting the $\gamma$-secretase complex. Indeed, if $A P P$ and $P S$ mutations reduce $\gamma$-secretase cleavage of APP, the proposed use of $\gamma$-secretase inhibitors as therapeutic agents in $\mathrm{AD}$ might lead to an unwanted enhancement of the neuronal degeneration.

Because of their dominant mode of inheritance and ability to increase the ratio of $\mathrm{A} \beta 42$ to $\mathrm{A} \beta 40$ in vitro [44], $A P P$ and $P S$ mutations are generally considered toxic gain-of-function alleles in the context of $\mathrm{AD}$ pathogenesis. However, when taking, for example, Notch signaling as a functional readout of PS or $\gamma$-secretase function, several studies suggest that AD-causing $P S$ mutations are intrinsically at least partial loss-of-function mutations. Indeed, loss of Notch cleavage or signaling has been demonstrated in mammalian cell lines $[60,61]$ and in Caenorhabditis elegans [62,63]. Further support for this idea comes from a strong loss-of-function mutation in the C. elegans $P S$ homologue sel-12 (C60S), isolated in a forward genetic screen [64], which corresponds to the human PS1 C92S mutation and is known to cause $\mathrm{AD}$ [65] by increasing the ratio of $\mathrm{A} \beta 42$ to $\mathrm{A} \beta 40$ [66,67]. Nevertheless, the situation is not completely clear because, although all $P S$ mutations and the partial reduction of normal PS1 activity [68] increase the ratio of A $\beta 42$ to $A \beta 40$, the total loss of PS results in loss of both $A \beta 40$ and $A \beta 42$ [45]. In addition, it is not well established if the increased $A \beta 42: A \beta 40$ ratio induced by the PS mutation is caused by a decrease in $\mathrm{A} \beta 40$, an increase in $\mathrm{A} \beta 42$ or a combination of both.

Interestingly, recent studies show that with respect to the generation of $A \beta$ peptides from $A P P, A D$-causing PS mutations have reliable loss-of-function properties. In a recently developed, highly reproducible cellular assay, we observed that all nine tested PS mutations consistently decreased $A \beta 40$ and accumulated direct $\gamma$-secretase substrates in the form of APP C-terminal fragments, a sign of decreased PS activity (S. Kumar-Singh et al., unpublished). Although the $\mathrm{A} \beta 42 \mathrm{~A} A \beta 40$ ratio was significantly increased for all, in only four PS mutations a significant increase in A $\beta 42$ was noted. A recent report on PS2 mutations has also shown A $\beta 40$ loss [69] and similar results were obtained using PS-deficient cells (B. De Strooper et al., unpublished). The interesting conclusion from these studies is that AD-related $P S$ mutations are less efficient in cleaving several $\gamma$-secretase substrates including APP and therefore behave as intrinsic biological partial loss-of-function alleles regarding $\gamma$-secretase function. Consistent with decreased $\gamma$-secretase activity for clinical $\mathrm{AD}$ mutations, a recent study has shown that both $P S$ and APP mutations located in the vicinity of the $\gamma$-secretase-cleavage site reduce $\gamma$-secretase-mediated liberation of the APP C-terminal fragment [70].

Together these results suggest that the consistently increased $\mathrm{A} \beta 42 \mathrm{~A} \beta 40$ ratios induced by $\mathrm{AD}$-causing $A P P$ and $P S$ mutations are the consequence of reduced $\gamma$-secretase activity. However, the exact mechanism is not understood.

\section{Is there a role for loss of PS in amyloid-independent neurodegeneration?}

With respect to this gain- versus loss-of-function discussion, interesting results have come from several recent studies. Conditional knockout mice lacking both PS in the post-natal forebrain showed progressive synaptic impairments and, importantly, severe age-dependent neurodegeneration characterized by cytoplasmic accumulations of hyperphosphorylated tau [71,72] but no $\mathrm{A} \beta$ deposits [73]. Because these results show that complete loss of PS can lead to an amyloid-independent form of tau-positive 
neurodegeneration, recent findings of PS mutations in FTD become highly intriguing. It was suggested that the PS1 G183V mutation, which is associated with FTD and with tauopathy in the form of Pick's disease but not with $\mathrm{A} \beta$ plaques, might also have loss-of-function properties because it affects the splice signal at the junction of the sixth exon and intron [57]. Interestingly, follow-up studies indeed revealed that this mutation, in addition to producing full-length PS1 G183V protein, also generates alternative transcripts that either lack exon six or exons six and seven leading to truncated proteins. In addition, cellular $\gamma$-secretase assays show that the truncated proteins behave as complete null alleles also suggesting a loss-of-function mechanism (Dermaut et al., unpublished). Strikingly, a loss-of-function mechanism has also been proposed for the PS1 insArg352 mutation that strongly inhibits $\gamma$-secretase cleavage of both Notch and APP [74] and is associated with FTD [56] without amyloid pathology (B. Boeve, personal communication).

Although additional confirmation is needed to establish a role of PS in amyloid-independent FTD, these studies suggest that throughout the PS spectrum of disorders, ranging from $\mathrm{AD}$ with a strong amyloid component to possibly tau-positive AD [57], partial PS loss-of-function might be the common theme, an idea that is also supported by studies suggesting that AD-risk-increasing alleles in the regulatory region of PS1 significantly decrease PS expression levels $[75,76]$. In addition, several recent cell biological studies have suggested that $P S$ mutations through reduced PI3K-Akt signaling promote glycogen synthase kinase $3 \beta$ (GSK3 $\beta$ ) activity and hence tau hyperphosphorylation [77-79], further suggesting a direct mechanistic link between PS loss-of-function and tau pathology.

Another line of recent evidence has linked dysfunction of the endosomal-lysosomal degradative system to loss of PS function $[80,81]$. This is interesting, because neurodegeneration is a frequent observation in lysosomal disorders [82] and abnormalities in the endosomal-lysosomal system have long been thought to be an early and prominent feature in $\mathrm{AD}$ [83]. In addition, tauopathy in the form of tau tangles is a highly consistent feature in the lysosomal disorder Niemann-Pick Type C [84] that, in its adult onset form, can present with FTD-like dementia. Strikingly, the recent finding that mutations in the charged multivesicular body protein 2B (CHMBP2B) on chromosome 3 cause FTD [85] further implicates dysfunctional late-endosomal or lysosomal activity in neuronal degeneration.

\section{Is tau the central molecule in the AD-FTD spectrum?}

When placed in the context of previous genotypephenotype correlation studies, recent findings show that FTD and AD not only share important clinical and neuropathological features but are also etiologically linked at the molecular genetic level, implying that these disorders are part of a genetically interconnected spectrum of presenile degenerative brain disorders. In addition, recent studies showing that: (i) genetic alterations at the level of MAPT are strongly associated with different types of tau-mediated neurodegeneration; (ii)

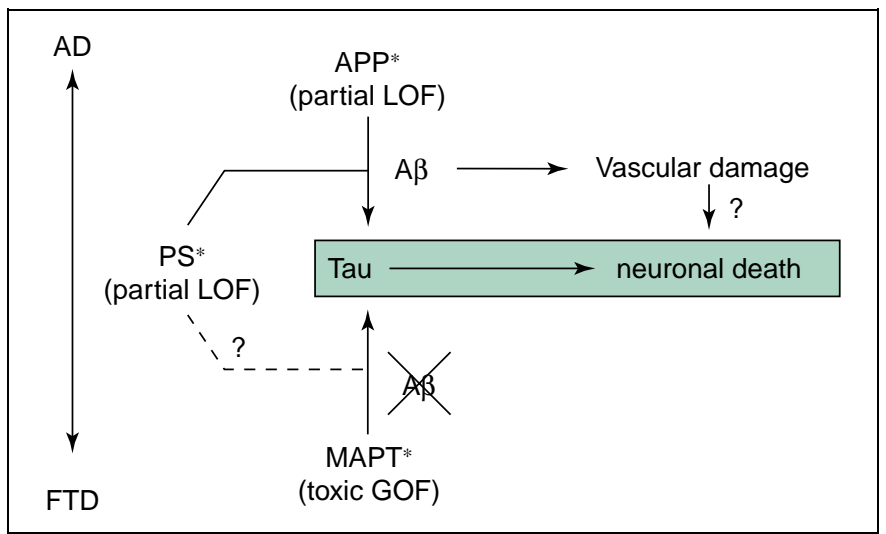

Figure 5. Tau is central in the AD-FTD spectrum. A speculative model for taumediated neurodegeneration in the genetic AD-FTD spectrum of brain disorders. The asterisks $\left(^{*}\right)$ refer to disease-associated genetic alterations in APP, PS and MAPT that, through gain- (GOF) or loss-of-function (LOF) mechanisms, result in taupositive neurodegeneration (shown in the center). The relative presence (shown in the upper panel) or absence (lower panel) of $A \beta$ pathology is indicated $(A \beta)$. The diagram shows that, in addition to tau pathology, APP and typical PS mutations lead to $A \beta$-positive pathology causing vascular damage and hence neuronal death. By contrast, genetic alterations in MAPT lead to A $\beta$-independent tau-mediated neuronal death. Recent evidence suggests that loss of PS function might result in $A \beta$-negative tau-mediated neurodegeneration (dashed line) further emphasizing that tau is the central molecule in the AD-FTD spectrum.

AD-causing $P S$ mutations are intrinsically loss-of-function alleles; and (iii) PS loss-of-function can lead to tau pathology, lead us to propose that tau is the major player throughout this AD-FTD spectrum (Figure 5). Within this framework, the further etiologic and mechanistic establishment of PS loss-of-function in amyloid-independent and tau-mediated neurodegeneration as well as the identification of the molecular defect leading to taunegative FTD (caused by MAPT or another gene nearby) are the most exciting and important future research topics.

\section{Acknowledgements}

Our research is supported by the Special Research Fund of the University of Antwerp, the Fund for Scientific Research Flanders (FWO-F), the Interuniversity Attraction Poles (IUAP) program P5/19 of the Belgian Science Policy Office (BELSPO), the International Alzheimer's Research Foundation (IARF) Belgium, the Alzheimer's Association USA and the EU contract LSHM-CT-2003-503330 (APOPIS). R.R. and M.C. are postdoctoral fellows of the FWO-F.

\section{References}

1 Goate, A. et al. (1991) Segregation of a missense mutation in the amyloid precursor protein gene with familial Alzheimers-disease. Nature 349, 704-706

2 Levy-Lahad, E. et al. (1995) Candidate gene for the chromosome-1 familial Alzheimers-disease locus. Science 269, 973-977

3 Sherrington, R. et al. (1995) Cloning of a gene bearing missense mutations in early-onset familial Alzheimers-disease. Nature 375, 754-760

4 Hutton, M. et al. (1998) Association of missense and $5^{\prime}$-splice-site mutations in tau with the inherited dementia FTDP-17. Nature 393, $702-705$

5 Poorkaj, P. et al. (1998) Tau is a candidate gene for chromosome 17 frontotemporal dementia. Ann. Neurol. 43, 815-825

6 Farrer, L.A. et al. (1997) Effects of age, sex, and ethnicity on the association between apolipoprotein $\mathrm{E}$ genotype and Alzheimer disease - a meta-analysis. J. Am. Med. Assoc. 278, 1349-1356

7 Saunders, A.M. et al. (1993) Apolipoprotein E4 is associated with lateonset familial and sporadic Alzheimers-disease. Am. J. Hum. Genet. $53,152-152$ 
8 Strittmatter, W.J. et al. (1993) Apolipoprotein-e - high-avidity binding to beta-amyloid and increased frequency of type-4 allele in late-onset familial Alzheimer-disease. Proc. Natl. Acad. Sci. U. S. A. 90, 1977-1981

9 Tanzi, R.E. and Bertram, L. (2005) Twenty years of the Alzheimer's disease amyloid hypothesis: a genetic perspective. Cell 120, 545-555

10 Wisniewski, K.E. et al. (1985) Occurrence of neuropathological changes and dementia of Alzheimer's disease in Down's syndrome. Ann. Neurol. 17, 278-282

11 Mann, D.M. (1988) The pathological association between Down syndrome and Alzheimer disease. Mech. Ageing Dev. 43, 99-136

12 Levy, E. et al. (1990) Mutation of the Alzheimers-disease amyloid gene in hereditary cerebral-hemorrhage, Dutch type. Science 248, 1124-1126

13 van Broeckhoven, C. et al. (1990) Amyloid-beta protein-precursor gene and hereditary cerebral-hemorrhage with amyloidosis (Dutch). Science 248, 1120-1122

14 Hendriks, L. et al. (1992) Presenile-dementia and cerebral-hemorrhage linked to a mutation at codon-692 of the $\beta$-amyloid precursor protein gene. Nat. Genet. 1, 218-221

15 Kumar-Singh, S. et al. (2002) Dense-core senile plaques in the Flemish variant of Alzheimer's disease are vasocentric. Am. J. Pathol. 161, 507-520

16 Mullan, M. et al. (1992) A pathogenic mutation for probable Alzheimer's disease in the APP gene at the N-terminus of betaamyloid. Nat. Genet. 1, 345-347

17 Kumar-Singh, S. et al. (2000) Nonfibrillar diffuse amyloid deposition due to a $\gamma^{42}$-secretase site mutation points to an essential role for $\mathrm{N}$-truncated $\mathrm{A} \beta_{42}$ in Alzheimer's disease. Hum. Mol. Genet. 9, 2589-2598

18 Kumar-Singh, S. et al. (2005) Dense-core plaques in Tg2576 and PSAPP mouse models of Alzheimer's disease are centered on vessel walls. Am. J. Pathol. 167, 527-543

19 Rademakers, R. et al. (2004) The role of tau (MAPT) in frontotemporal dementia and related tauopathies. Hum. Mutat. 24, 277-295

20 Lee, V.M. et al. (2001) Neurodegenerative tauopathies. Annu. Rev. Neurosci. 24, 1121-1159

21 Reed, L.A. et al. (2001) Phenotypic correlations in FTDP-17. Neurobiol. Aging 22, 89-107

22 Rademakers, R. et al. (2003) Tau (MAPT) mutation Arg406Trp presenting clinically with Alzheimer disease does not share a common founder in Western Europe. Hum. Mutat. 22, 409-411

23 Lendon, C.L. et al. (1998) Hereditary dysphasic disinhibition dementia - a frontotemporal dementia linked to 17q21-22. Neurology 50, 1546-1555

24 Rademakers, R. et al. (2002) Tau negative frontal lobe dementia at 17q21: significant finemapping of the candidate region to a $4.8 \mathrm{cM}$ interval. Mol. Psychiatry 7, 1064-1074

25 Rosso, S.M. et al. (2001) Familial frontotemporal dementia with ubiquitin-positive inclusions is linked to chromosome 17q2l-22. Brain 124, 1948-1957

26 Cruts, M. et al. (2005) Genomic architecture of human 17q21 linked to frontotemporal dementia uncovers a highly homologous family of low copy repeats in the tau region. Hum. Mol. Genet. 14, 1753-1762

27 Zhukareva, V. et al. (2001) Loss of brain tau defines novel sporadic and familial tauopathies with frontotemporal dementia. Ann. Neurol. 49, 165-175

28 Zhukareva, V. et al. (2003) Selective reduction of soluble tau proteins in sporadic and familial frontotemporal dementias: an international follow-up study. Acta Neuropathol. (Berl.) 105, 469-476

29 Taniguchi, S. et al. (2004) The neuropathology of frontotemporal lobar degeneration with respect to the cytological and biochemical characteristics of tau protein. Neuropathol. Appl. Neurobiol. 30, 1-18

30 Stefansson, H. et al. (2005) A common inversion under selection in Europeans. Nat. Genet. 37, 129-137

31 Stankiewicz, P. and Lupski, J.R. (2002) Molecular-evolutionary mechanisms for genomic disorders. Curr. Opin. Genet. Dev. 12, 312-319

32 Wittmann, C.W. et al. (2001) Tauopathy in Drosophila: neurodegeneration without neurofibrillary tangles. Science 293, 711-714

33 Santacruz, K. et al. (2005) Tau suppression in a neurodegenerative mouse model improves memory function. Science 309, 476-481
34 Morris, H.R. et al. (2001) The genetic and pathological classification of familial frontotemporal dementia. Arch. Neurol. 58, 1813-1816

35 Mann, D.M. et al. (2000) Molecular classification of the dementias. Lancet 355,626

36 Kertesz, A. (2003) Pick Complex: an integrative approach to frontotemporal dementia: primary progressive aphasia, corticobasal degeneration, and progressive supranuclear palsy. Neurologist 9, 311-317

37 Conrad, C. et al. (1997) Genetic evidence for the involvement of tau in progressive supranuclear palsy. Ann. Neurol. 41, 277-281

38 Baker, M. et al. (1999) Association of an extended haplotype in the tau gene with progressive supranuclear palsy. Hum. Mol. Genet. 8, 711-715

39 Houlden, H. et al. (2001) Corticobasal degeneration and progressive supranuclear palsy share a common tau haplotype. Neurology 56, 1702-1706

40 Kwok, J.B. et al. (2004) Tau haplotypes regulate transcription and are associated with Parkinson's disease. Ann. Neurol. 55, 329-334

41 Pittman, A. M. et al. (2005) Linkage disequilibrium fine-mapping and haplotype association analysis of the tau gene in progressive supranuclear palsy and corticobasal degeneration. J. Med. Genet., DOI: 10. 1136/jmg.2005.031377

42 Hutton, M. et al. (2001) Analysis of tauopathies with transgenic mice. Trends Mol. Med. 7, 467-470

43 Jackson, G.R. et al. (2002) Human wild-type tau interacts with wingless pathway components and produces neurofibrillary pathology in Drosophila. Neuron 34, 509-519

44 Citron, M. et al. (1997) Mutant presenilins of Alzheimer's disease increase production of 42-residue amyloid $\beta$-protein in both transfected cells and transgenic mice. Nat. Med. 3, 67-72

45 De Strooper, B. et al. (1998) Deficiency of presenilin-1 inhibits the normal cleavage of amyloid precursor protein. Nature 391, 387-390

46 Wolfe, M.S. et al. (1999) Two transmembrane aspartates in presenilin1 required for presenilin endoproteolysis and gamma-secretase activity. Nature 398, 513-517

47 Lippa, C.F. et al. (2000) Familial Alzheimer's disease: site of mutation influences clinical phenotype. Ann. Neurol. 48, 376-379

48 Mann, D.M.A. et al. (2001) Amyloid angiopathy and variability in amyloid beta deposition is determined by mutation position in presenilin-1-linked Alzheimer's disease. Am. J. Pathol. 158, 2165-2175

49 Dermaut, B. et al. (2001) Cerebral amyloid angiopathy is a pathogenic lesion in Alzheimer's disease due to a novel presenilin 1 mutation. Brain 124, 2383-2392

50 Nochlin, D. et al. (1998) Amyloid angiopathy in a Volga German family with Alzheimer's disease and a presenilin-2 mutation (N141I). Ann. Neurol. 43, 131-135

51 Prihar, G. et al. (1999) Alzheimer disease PS-1 exon 9 deletion defined. Nat. Med. 5, 1090

52 Crook, R. et al. (1998) A variant of Alzheimer's disease with spastic paraparesis and unusual plaques due to deletion of exon 9 of presenilin 1. Nat. Med. 4, 452-455

53 Houlden, H. et al. (2000) Variant Alzheimer's disease with spastic paraparesis and cotton wool plaques is caused by PS-1 mutations that lead to exceptionally high amyloid- $\beta$ concentrations. Ann. Neurol. 48, 806-808

54 Hutton, M. (2004) Presenilin mutations associated with frontotemporal dementia. Ann. Neurol. 55, 604-606

55 Raux, G. et al. (2000) Dementia with prominent frontotemporal features associated with L113P presenilin 1 mutation. Neurology 55, 1577-1578

56 Tang-Wai, D. et al. (2002) Familial frontotemporal dementia associated with a novel presenilin-1 mutation. Dement. Geriatr. Cogn. Disord. 14, 13-21

57 Dermaut, B. et al. (2004) A novel presenilin 1 mutation associated with Pick's disease but not $\beta$-amyloid plaques. Ann. Neurol. 55, 617-626

58 Ikeda, M. et al. (1996) The clinical phenotype of two missense mutations in the presenilin I gene in Japanese patients. Ann. Neurol. 40, 912-917 
59 Halliday, G.M. et al. (2005) Pick bodies in a family with presenilin-1 Alzheimer's disease. Ann. Neurol. 57, 139-143

60 Schroeter, E.H. et al. (2003) A presenilin dimer at the core of the $\gamma$-secretase enzyme: insights from parallel analysis of Notch 1 and APP proteolysis. Proc. Natl. Acad. Sci. U. S. A. 100, 13075-13080

61 Song, W.H. et al. (1999) Proteolytic release and nuclear translocation of Notch-1 are induced by presenilin-1 and impaired by pathogenic presenilin-1 mutations. Proc. Natl. Acad. Sci. U. S. A. 96, 6959-6963

62 Baumeister, R. et al. (1997) Human presenilin-1, but not familial Alzheimer's disease (FAD) mutants, facilitate Caenorhabditis elegans Notch signalling independently of proteolytic processing. Genes Funct. 1, 149-159

63 Levitan, D. et al. (1996) Assessment of normal and mutant human presenilin function in Caenorhabditis elegans. Proc. Natl. Acad. Sci. U. S. A. 93, 14940-14944

64 Levitan, D. and Greenwald, I. (1995) Facilitation of lin-12-mediated signalling by sel-12, a Caenorhabditis elegans S182 Alzheimer's disease gene. Nature 377, 351-354

65 Tedde, A. et al. (2003) Identification of new presenilin gene mutations in early-onset familial Alzheimer disease. Arch. Neurol. 60, 1541-1544

66 Lewis, P.A. et al. (2000) The presenilin 1 C92S mutation increases $\alpha \beta$ 42 production. Biochem. Biophys. Res. Commun. 277, 261-263

67 Okochi, M. et al. (2000) A loss of function mutant of the presenilin homologue SEL-12 undergoes aberrant endoproteolysis in Caenorhabditis elegans and increases $\alpha \beta 42$ generation in human cells. J. Biol. Chem. 275, 40925-40932

68 Refolo, L.M. et al. (1999) Antisense-induced reduction of presenilin 1 expression selectively increases the production of amyloid $\beta 42$ in transfected cells. J. Neurochem. 73, 2383-2388

69 Walker, E.S. et al. (2005) Presenilin 2 familial Alzheimer's disease mutations result in partial loss of function and dramatic changes in $\alpha \beta$ 42/40 ratios. J. Neurochem. 92, 294-301

70 Wiley, J.C. et al. (2005) Familial Alzheimer's disease mutations inhibit gamma-secretase-mediated liberation of beta-amyloid precursor protein carboxy-terminal fragment. J. Neurochem. 94, 1189-1201

71 Feng, R. et al. (2004) Forebrain degeneration and ventricle enlargement caused by double knockout of Alzheimer's presenilin-1 and presenilin-2. Proc. Natl. Acad. Sci. U. S. A. 101, 8162-8167
72 Saura, C.A. et al. (2004) Loss of presenilin function causes impairments of memory and synaptic plasticity followed by agedependent neurodegeneration. Neuron 42, 23-36

$73 \mathrm{Yu}, \mathrm{H}$. et al. (2001) APP processing and synaptic plasticity in presenilin-1 conditional knockout mice. Neuron 31, 713-726

74 Amtul, Z. et al. (2002) A presenilin 1 mutation associated with familial frontotemporal dementia inhibits $\gamma$-secretase cleavage of APP and notch. Neurobiol. Dis. 9, 269-273

75 Lambert, J.C. et al. (2001) The $-48 \mathrm{C} / \mathrm{T}$ polymorphism in the presenilin 1 promoter is associated with an increased risk of developing Alzheimer's disease and an increased A $\beta$ load in brain. J. Med. Genet. 38, 353-355

76 Theuns, J. et al. (2003) Alzheimer-associated C allele of the promoter polymorphism $-22 \mathrm{C}>\mathrm{T}$ causes a critical neuron-specific decrease of presenilin 1 expression. Hum. Mol. Genet. 12, 869-877

77 Baki, L. et al. (2004) PS1 activates PI3K thus inhibiting GSK-3 activity and tau overphosphorylation: effects of FAD mutations. EMBO J. 23, 2586-2596

78 Kang, D.E. et al. (2005) Presenilins mediate PI3K/Akt and ERK activation via select signaling receptors: selectivity of PS2 in PDGF signaling. J. Biol. Chem., DOI:10.1074/jbc.M500833200

79 Ryder, J. et al. (2004) Akt/GSK3beta serine/threonine kinases: evidence for a signalling pathway mediated by familial Alzheimer's disease mutations. Cell. Signal. 16, 187-200

80 Esselens, C. et al. (2004) Presenilin 1 mediates the turnover of telencephalin in hippocampal neurons via an autophagic degradative pathway. J. Cell Biol. 166, 1041-1054

81 Wilson, C.A. et al. (2004) Degradative organelles containing mislocalized $\alpha$ and $\beta$-synuclein proliferate in presenilin-1 null neurons. J. Cell Biol. 165, 335-346

82 Walkley, S.U. (1998) Cellular pathology of lysosomal storage disorders. Brain Pathol. 8, 175-193

83 Nixon, R.A. et al. (2001) The neuronal endosomal-lysosomal system in Alzheimer's disease. J. Alzheimers Dis. 3, 97-107

84 Auer, I.A. et al. (1995) Paired helical filament tau (PHFtau) in Niemann-Pick type $\mathrm{C}$ disease is similar to PHFtau in Alzheimer's disease. Acta Neuropathol. (Berl.) 90, 547-551

85 Skibinski, G. et al. (2005) Mutations in the endosomal ESCRTIIIcomplex subunit CHMP2B in frontotemporal dementia. Nat. Genet. $37,806-808$

\title{
Articles of interest from Current Opinion in Genetics and Development
}

\author{
Genomic views of chromatin \\ Dana J. Huebert and Bradley E. Bernstein \\ Current Opinion in Genetics and Development 15, 476-481
}

Delving into the diversity of facultative heterochromatin: the epigenetics

of the inactive $X$ chromosome

Edith Heard

Current Opinion in Genetics and Development 15, 482-489

DNA methylation and histone modifications: teaming up to silence genes François Fuks

Current Opinion in Genetics and Development 15, 490-495

How microRNAs control cell division, differentiation and death

Eric A. Miska

Current Opinion in Genetics and Development 15, 563-568

HMG proteins: dynamic players in gene regulation and differentiation

Marco E. Bianchi and Alessandra Agresti

Current Opinion in Genetics and Development 15, 496-506 